\title{
Comportement du Gonflement d'un Polymère Superabsorbant vis-à-vis de l'Eau dans un Sol Sableux
}

\author{
H.Bahaj ${ }^{a}$, R.Benaddi ${ }^{a}$, M.Bakass $^{a}$, C.Bayane ${ }^{a}$ \& J.P. Bellat ${ }^{b}$ \\ a Laboratoire de Recherche sur la Réactivité des Matériaux et l'Optimisation des Procédés, REMATOP, \\ Faculté des Sciences Semlalia, Université Cadi Ayyad, B.P. 2390 Marrakech (Maroc). \\ baboratoire de Recherche sur la Réactivité des Solides, UMR 5613 CNRS, Université de Bourgogne, UFR \\ ST, av. A. Savary, B.P. 47870, F-21078 Dijon cedex, France \\ bahaj_hanane@yahoo.fr, r.benaddi@hotmail.fr, bakass@ucam.ac.ma,c.bayane@ucam.ac.ma, \\ jean-pierre.bellat@u-bourgogne.fr
}

\begin{abstract}
Résumé
Cette étude concerne un polymère superabsorbant capable de retenir entre ces chaînes macromoléculaires 300 fois sa masse en eau. Cette intéressante attitude à se gonfler a été évaluée en fonction de plusieurs paramètres. Les résultats obtenus ont montré que le gonflement du polymère $\mathrm{X} 3$ dépend de la valeur du pH de la solution absorbée, mais l'influence de la température de la solution absorbée ne devient perceptible qu'à partir des valeurs supérieures à $40^{\circ} \mathrm{C}$. L'exame $n$ de l'effet de la présence des contres ions sur la capacité d'absorption maximale a permis de conclure que la présence des cations mono ou divalents dans la solution absorbée entraîne une réduction du taux d'absorption maximal du polymère au fur et à mesure que la concentration en cations augmente. Dans l'optique d'une future application de ce matériau dans l'agriculture, une partie de ce travail a été consacrée à l'évaluation du phénomène du gonflement du polymère X3 dans un sol sableux pour différentes fractions et dispositions du polymère dans le sol.
\end{abstract}

\section{Mots clés:}

Polymère, Superabsorbant, Sol sableux, Absorption, Gonflement, pH, Température, Contres ions.

\section{INTRODUCTION}

Les polymères superabsorbants (type polyacrylates) sont des matériaux hydrophiles capables d'emmagasiner de grandes quantités d'eau entre leurs chaînes macromoléculaires. En raison de cette caractéristique, ils sont utilisés dans plusieurs domaines tels que ceux de l'horticulture et de l'agriculture [1,2]. Les polymères superabsorbants trouvent leur application également dans l'extraction, par complexation dans les solutions aqueuses, de certains cations métalliques tels que le mercure, le plomb et le cadmium [3]. De nombreuses études ont été publiées sur le gonflement des polyacrylates en solution. En fait, cette propriété dépend de plusieurs facteurs à savoir le degré de réticulation, le degré d'ionisation, la quantité d'initiateur et la quantité du monomère [4,5]. Le gonflement d'un polymère superabsorbant dépend aussi de certains paramètres physico-chimiques tels que la température, le $\mathrm{pH}$ du milieu, la composition et la concentration en éléments chimiques de la solution absorbée [6-8]. L'étude des mélanges constitués du sol et du gel a montré que la capacité d'absorption d'eau est fonction d'une part de la quantité du gel dans le sol et d'autre part du type du polymère employé pour préparer le gel [4]. L'application des gels dans des sols sableux permet d'augmenter la quantité d'eau retenue par le sol [9]. Elle permet également de retenir certains cations utiles pour la croissance des plantes [1]. Ainsi l'introduction des gels dans les sols peut améliorer les conditions d'établissement et de croissance des plantes $[1,10]$.

\section{TECHNIQUES EXPERIMENTALES}

\subsection{Mesure de la capacité d'absorption}

Pour mesurer la capacité d'absorption maximale du polymère ou du mélange polymère - sol, une masse initiale connue d'échantillon a été introduite dans un tube. On ajoute progressivement de l'eau jusqu'à apparition et maintien d'eau à la surface de l'échantillon. Ainsi l'excès d'eau est séché à l'aide du papier filtre. Connaissant la masse de l'échantillon sec et celle de l'échantillon saturé en eau, on peut calculer le taux d'absorption maximal en utilisant la relation suivante:

$$
Q_{H_{2} O}=\frac{m_{2}-m_{1}}{m_{1}}
$$

Avec $m_{1}$ : la masse de l'échantillon sec.

$\mathrm{m}_{2}$ : la masse de l'échantillon saturé en eau.

Qн2о: le taux d'absorption maximal (grammes d'eau par grammes du polymère).

\subsection{Echantillon}

Le polymère en cours d'étude est un polyélectrolyte superabsorbant de couleur blanche commercialisé sous la référence NORSOLOR CAS 25608-12-2. II est préparé à partir de l'acide acrylique. II est capable de retenir jusqu'à 300 fois son poids en eau. II se présente sous forme de billes sphériques fortement déformées, d'aspect surfacique non poreux et dont le diamètre varie entre 100 à $500 \mu \mathrm{m}$. L'étude de la stabilité thermique de ce polymère par analyse

This is an Open Access article distributed under the terms of the Creative Commons Attribution-Noncommercial License (http://creativecommons.org/licenses/by-nc/3.0/), which permits unrestricted use, distribution, and reproduction in any noncommercial medium, provided the original work is properly cited. 
thermogravimétrique a montré que celui-ci est stable jusqu'à une température de $180^{\circ} \mathrm{C}$ et qu'il présente trois ty pes de pertes d'eau. La figure 1 illustre la perte de masse du polymère $\mathrm{X} 3$ en fonction de la température La première perte est observée entre 42 et $57^{\circ} \mathrm{C}$, elle peut être attri buée à une perte d'eau surfacique. La deuxième perte commence vers $67^{\circ} \mathrm{C}$ et se termine vers $9^{\circ} \mathrm{C}$, peut être due à l'éli mination de l'eau moyennement liée aux chaînes macromoléculaires. La dernière perte est apparue entre 127 et $147^{\circ} \mathrm{C}$. Cell e-ci a été attribuée à l'élimination de l'eau fortement liée aux chaînes polymériques. Le produit final garde sa couleur blanche initiale. Au delà d'une température de $180^{\circ} \mathrm{C}$ l'augme ntation de la température provoque la dégradation du polymère via la destruction de la matière organique.

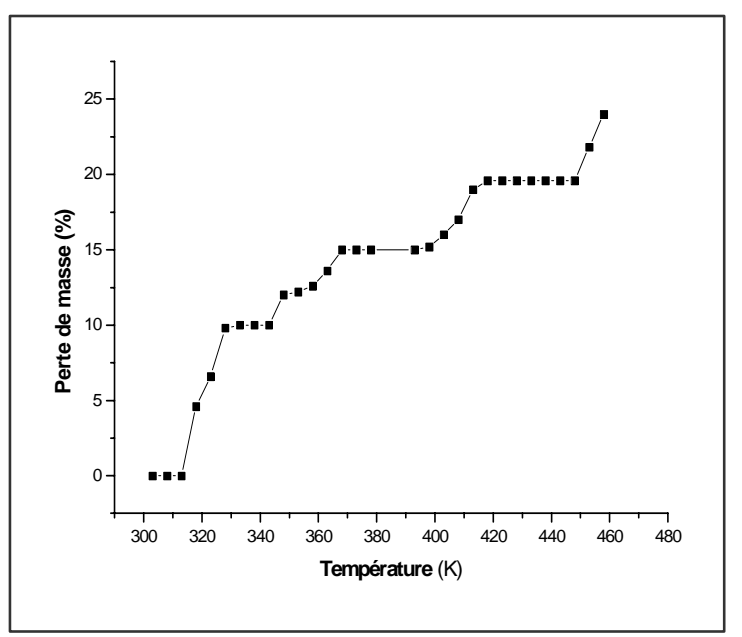

Figure 1: Evolution de la perte de masse en fonction de la température.

Les images du polymère anhydre obtenues par microscope électronique à balayage type JSM-6400 sont représentées sur la figure 2. Le sol employé dans cette étude est un sol sableux granulé de formule chimique $\mathrm{SiO}_{2}$, dont les dimensions des grains varient entre 0,25 et $0,30 \mathrm{~mm}$.

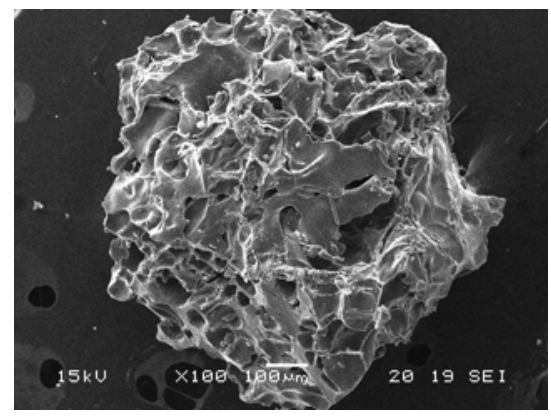

A

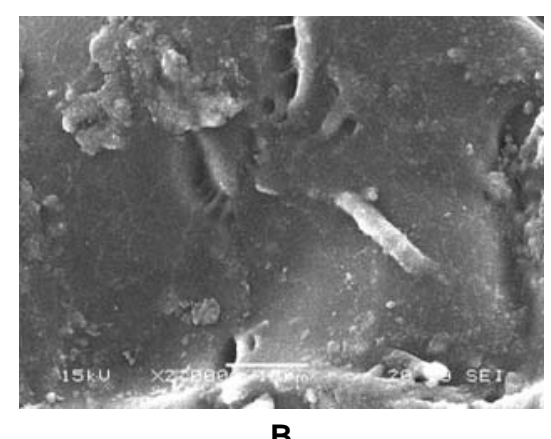

Figure 2: Vue au microscope électronique à balayage du polymère $X 3$ anhydre avec $(A) \times 100 ;(B) \times 2000$

\section{RESULTATS EXPERIMENTAUX}

\subsection{Effet de la disposition}

L'étude de l'effet de la disposition du polymère sur le taux de rétention de l'eau par un sol sableux a été réalisée à température ambiante et pression atmosphérique. En fait, cette étude a été effectuée pour différentes fractions du polymère dans le sol $(0,2 ; 0,5$ et $1 \%)$. la figure 3 illustre la variation de la capacité d'absorption maximale du système polymère - sol pour chaque pourcentage et disposition du polymère vis-à-vis du sol.

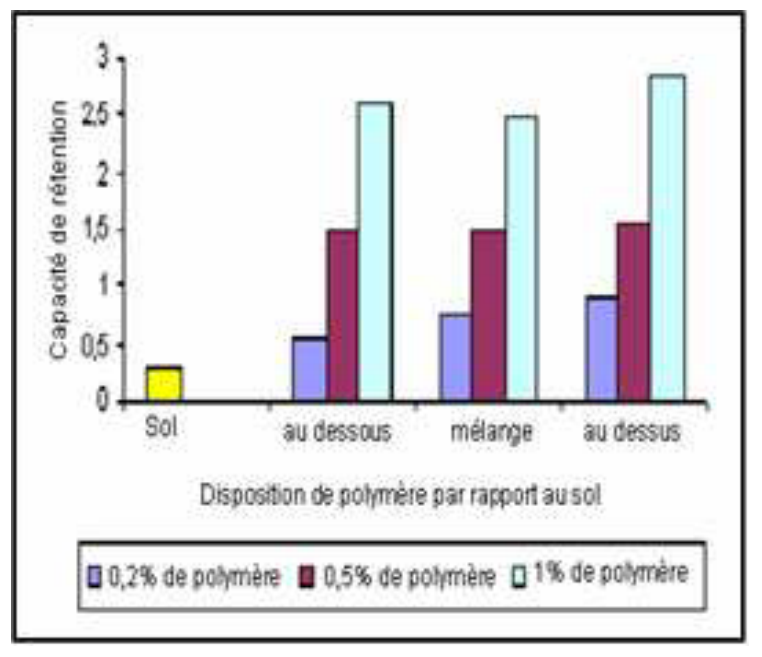

Figure 3 : Evolution du taux de saturation des mélanges polymère - sol en fonction des pourcentages du polymère et de sa disposition vis-à-vis du sol.

D'après cette figure, on peut constater que la présence du polymère dans le sol permet d'augmenter la capacité de rétention d'eau par le sol. En effet, le sol seul peut retenir jusqu'à 0,28 fois sa masse en eau, cette valeur croît considérablement en présence du polymère au fur et à mesure que le pourcentage en celui-ci augmente et ceci quelle que soit sa disposition. Pour des pourcentages en polymère de 0,5 et $1 \%$, la quantité d'eau retenue par gramme du sol est plus importante en comparaison avec celle retenue dans le cas d'un pourcentage de $0,2 \%$ en polymère. Dans le cas d'un tel pourcentage $(0,2 \%)$ du polymère dans le sol et pour une disposition du polymère au dessus du sol, la quantité d'eau retenue par le système est supérieure à celle retenue dans le cas où le polymère est mélangé ou mis au dessous du sol. Ceci peut être expliqué par un effet de masse. En fait, lorsque le polymère est placé au dessous ou mélangé avec le sol, on assiste d'un côté à une gêne de la diffusion de l'eau dans l'espace intergranulaire entravant par voie de conséquence le transport de l'eau jusqu'au polymère. D'un autre côté l'intensité des répulsions de natures électrostatiques entre les chaînes macromoléculaires, responsable du gonflement, est insuffisante pour repousser les grains du sol sableux et permettre au gel de se gonfler librement. Ainsi, la capacité d'absorption du polymère se réduit. La figure 3 montre aussi qu'à partir d'un pourcentage du polymère supérieur ou égal à $0,5 \%$, la capacité de rétention d'eau par le système (polymère - sol) reste pratiquement constante quelle que soit la disposition du polymère par rapport au sol. En effet, l'usage des pourcentages importants du polymère dans le sol, et par conséquent un nombre de fonctions acides ionisables plus grand peut entraîner des forces de répulsions de natures électrostatiques de plus en plus significatives. Ainsi, dans le 
cas d'un pourcentage élevé en polymère, l'intensité engendrée par les répulsions entre les groupes carboxylates peut se contrarier à la compacité exercée par le sol sur le polymère, surtout s'il mis au dessous ou mélangé avec le sol. Ainsi le polymère peut repousser les grains du sol et se gonfler librement. L'histogramme donné par la figure 3, montre également que le taux de rétention d'eau maximum est obtenu pour un pourcentage de $1 \%$ du polymère. Etant donné que la qualité de vie des plantes dépend de la capacité de rétention de l'eau et des éléments nutritives par le sol, spécialement dans les zones arides et semi arides, on peut dire que la présence du polymère en cours d'étude dans le sol peut améliorer considérablement la qualité de vie des plantes par réduction éventuelle des pertes d'eau par infiltration dans le sol.

\subsection{Effet du pH du milieu}

L'étude de l'influence du $\mathrm{pH}$ du milieu sur le gonflement du polymère $X 3$ seul a été effectuée à pression atmosphérique et température ambiante. Le $\mathrm{pH}$ de la solution absorbée est amené à la valeur désirée par ajout d'une solution de $\mathrm{HCl}$ ou de $\mathrm{NaOH}$. La figure 4 illustre la variation de la capacité d'absorption maximale du polymère $\mathrm{X} 3$ en fonction du $\mathrm{pH}$ de la solution absorbée.

L'analyse de la figure 4 permet de mettre en évidence la variation du taux du gonflement maximum qui diffère complètement suivant la valeur du pH imposée.

- Le premier changement du taux d'absorption correspond à des valeurs $\mathrm{du} \mathrm{pH} \leq 3$, où on assiste à un accroissement rapide du taux d'absorption du polymère au fur et à mesure que la valeur du $\mathrm{pH}$ augmente pour atteindre une valeur de 250 fois sa masse en eau. Le fort accroissement que subit le système traduit l'hyper sensibilité du phénomène du gonflement vis-à-vis du $\mathrm{pH}$.

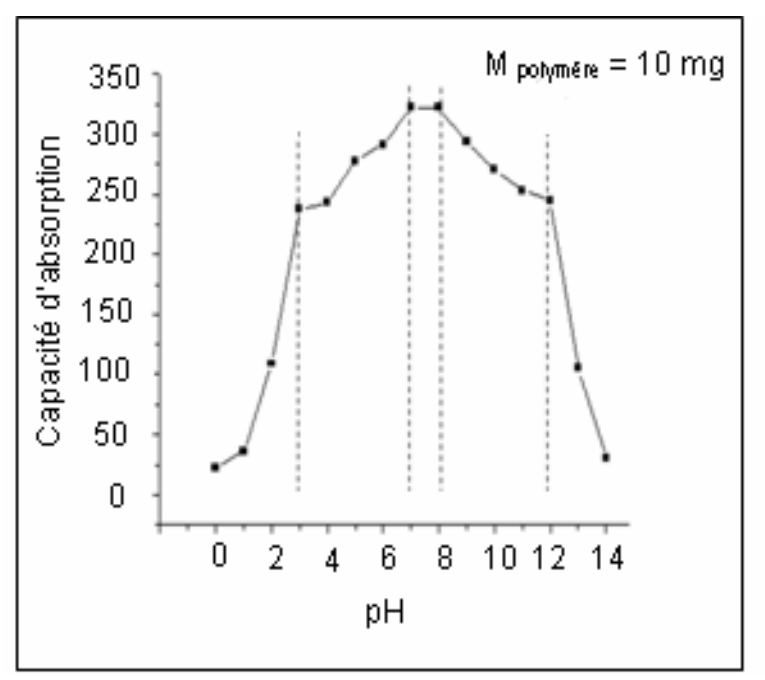

Figure 4 : Influence du $\mathrm{pH}$ du milieu sur le gonflement du polymère à $\mathrm{T}=25^{\circ} \mathrm{C}$.

- Pour des valeurs du pH comprises entre 3 et 7 , la capacité d'absorption du polymère continue à accroître mais d'une façon moins rapide jusqu'à une valeur de 300 fois sa masse en eau. En fait, ce résultat peut être expliqué par le fait que l'accroissement de la valeur du pH entraîne l'augmentation de nombres de fonctions acides ionisées et par conséquent les répulsions de natures électrostatiques entre sites chargés deviennent de plus en plus significatives. Ainsi, les chaînes macromoléculaires deviennent plus flexibles, favorisant davantage l'expansion du réseau polymérique, de ce fait le taux d'absorption augmente au fur et à mesure que la valeur du pH croît.

- Pour des valeurs du pH comprises entre 7 et 8 , le taux d'absorption demeure presque constant. Ce résultat peut être dû à l'usage de la soude pour ramener la valeur du $\mathrm{pH}$ de la solution absorbée à des valeurs supérieures à 7 . Les cations $\mathrm{Na}^{+}$ajoutés peuvent jouer un effet d'écran entre les sites chargés. En effet, les cations $\mathrm{Na}^{+}$peuvent s'insérer entre les macromolécules et réduire les répulsions de natures électrostatiques entre les groupes carboxylates et par conséquent la capacité du gonflement. On assiste donc à une compensation entre l'effet de l'augmentation de la valeur $\mathrm{du} \mathrm{pH}$ favorisant l'ionisation des fonctions acides et l'effet d'écran, induit par l'ion sodium, réduisant l'intensité des forces de répulsions de natures électrostatiques entre les sites chargés.

- Pour des valeurs du pH supérieures à 8, on assiste à une diminution progressive du taux d'absorption du polymère $\mathrm{X} 3$ jusqu'à une valeur du $\mathrm{pH}=12$, à partir de cette valeur du $\mathrm{pH}$, la capacité d'absorption subit une chute brusque au fur et à mesure que la valeur $\mathrm{du} \mathrm{pH}$ de la solution absorbée croît. Ce qui peut être expliqué par le fait que l'effet d'écran dû à l'ion de sodium provenant de l'ajout de la soude l'emporte sur l'effet d'ionisation des fonctions acides dû à l'accroissement de la valeur du $\mathrm{pH}$ de la solution absorbée. Un comportement presque similaire a été obtenu lors de l'étude de l'effet de certains paramètres physico-chimiques sur le gonflement d'un polyélectrolyte superabsorbant X10.

\subsection{Effet de la température}

L'étude suivante porte également sur l'évaluation de l'influence de la température de la solution absorbée sur l'aptitude du gonflement du polymère $\mathrm{X} 3$. Ceci a été réalisé pour différentes valeurs du $\mathrm{pH}(2,7$ et 10).

La figure 5 illustre la variation de la capacité d'absorption maximale du polymère $X 3$ en fonction de la température. L'analyse de cette figure montre que quelque soit la valeur $\mathrm{du} \mathrm{pH}$ de la solution absorbée, le taux d'absorption demeure presque constant pour des valeurs de températures comprises entre 10 et $40^{\circ} \mathrm{C}$. Au-delà de cette température, la capacité d'absorption diminue au fur et à mesure que la température de la solution absorbée croît et ce quelque soit le $\mathrm{pH}$ de cette solution.

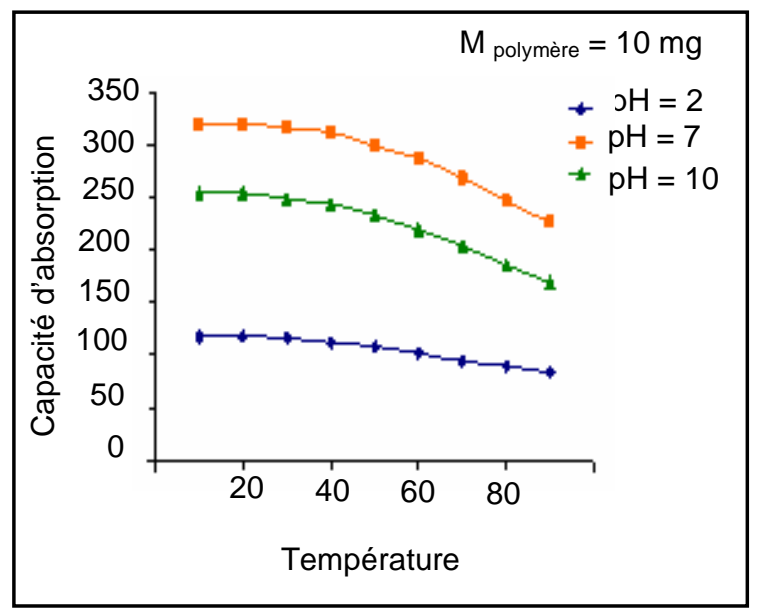

Figure 5: Effet de la température sur le gonflement du polymère.

Ces résultats peuvent être expliqués par la sensibilité des chaînes macromoléculaires à l'égard de la température. En 
effet, l'accroissement de la température peut affecter les propriétés élastiques des chaînes macromoléculaires. Ces dernières, sous l'effet de la température peuvent se dilater et devenir incapables de retenir les molécules de l'eau entre elles. En réalité à $\mathrm{pH}=7$ ou à $\mathrm{pH}=10$, les fonctions acides sont ionisées et les répulsions entre charges de même signe entraînent le gonflement du polymère. Ainsi l'élévation de la température favorise l'éloignement des chaînes macromoléculaires les unes par rapport aux autres. Et de ce fait provoque le départ de l'eau libre présent dans le réseau polymérique [11] et réduit par conséquent le taux d'absorption. Par contre à $\mathrm{pH}=2$, l'excès des ions hydroniums ajoutés déplace l'équilibre physico-chimique dans le sens de la protonation des groupes carboxylates. Ainsi, les chaînes macromoléculaires sont plus serrées et de ce fait moins sensible à l'élévation de la température

\subsection{Effet de la présence des cations}

Dans la même optique d'évaluation de l'effet des conditions du milieu sur le gonflement du polymère X3. Cette partie du travail concerne l'examen de l'effet de la présence de certains cations mono et divalents sur la capacité d'absorption maximale du polymère $\mathrm{X} 3$, et ce pour des valeurs du $\mathrm{pH}$ de 2, 7 et 10. Pour cette étude nous avons utilisé les solutions des sels suivantes : $\mathrm{NaCl}, \mathrm{KCl}, \mathrm{NH}_{4} \mathrm{Cl}$, $\mathrm{CaCl}_{2}$ et $\mathrm{MgCl}_{2}$ ayant tous en commun le même anion chlorure. Les figures 6, 7 et 8 présentent la variation de la capacité d'absorption en fonction de la concentration en cations de la solution absorbée.

Ces courbes montrent que la capacité d'absorption maximale du polymère $\mathrm{X} 3$ décroît au fur et à mesure avec l'accroissement de la concentration de la solution en cations et ce quelle que soit la valeur du $\mathrm{pH}$. Cette diminution peut être due à l'insertion des contres ions entre les chaînes macromoléculaires provoquant ainsi un effet d'écran sur les groupes carboxylates, $\mathrm{Ce}$ qui réduit l'intensité des répulsions électrostatiques entre les charges négatives. Cette insertion peut également provoquer une réticulation supplémentaire des chaînes macromoléculaires.

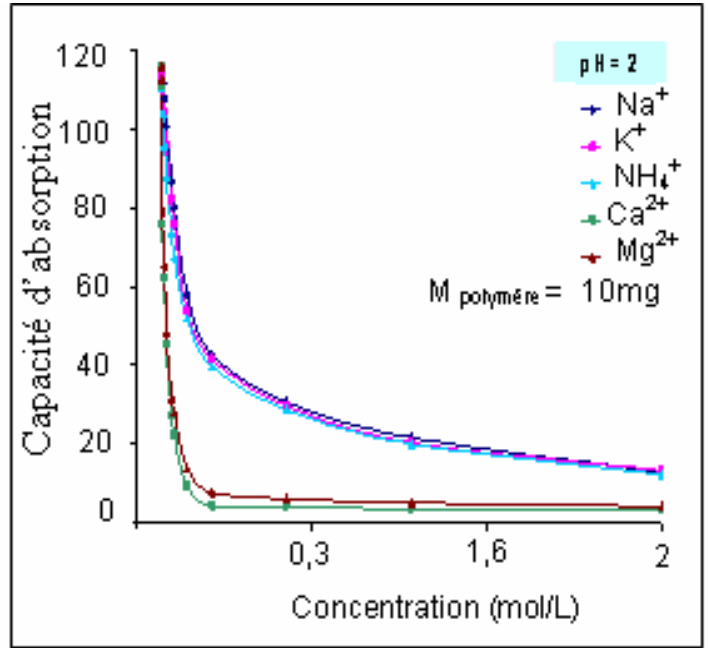

Figure 6 : Variation de la capacité d'absorption du polymère en fonction de la concentration de la solution adsorbée à $\mathrm{pH}=7$.

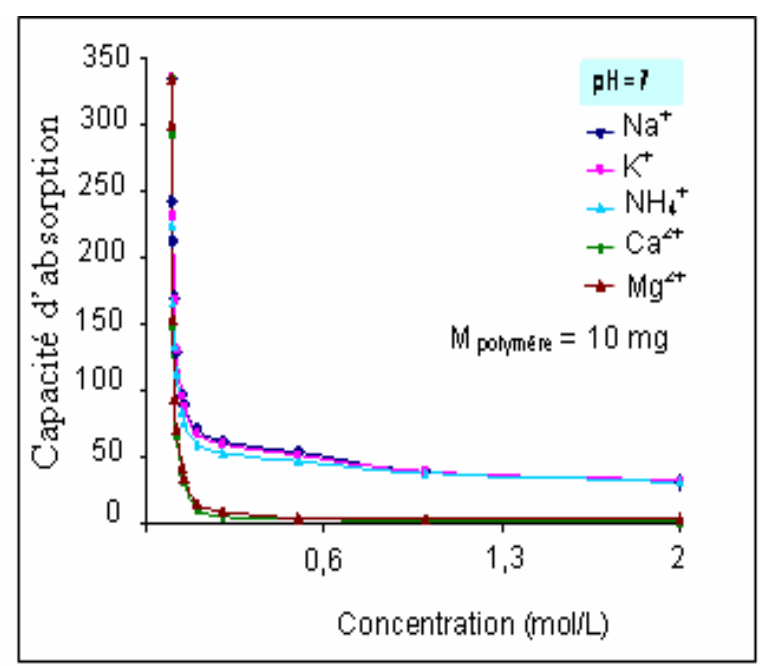

Figure 7 : Variation de la capacité d'absorption du polymère en fonction de la concentration de la solution adsorbée à $\mathrm{pH}=7$.

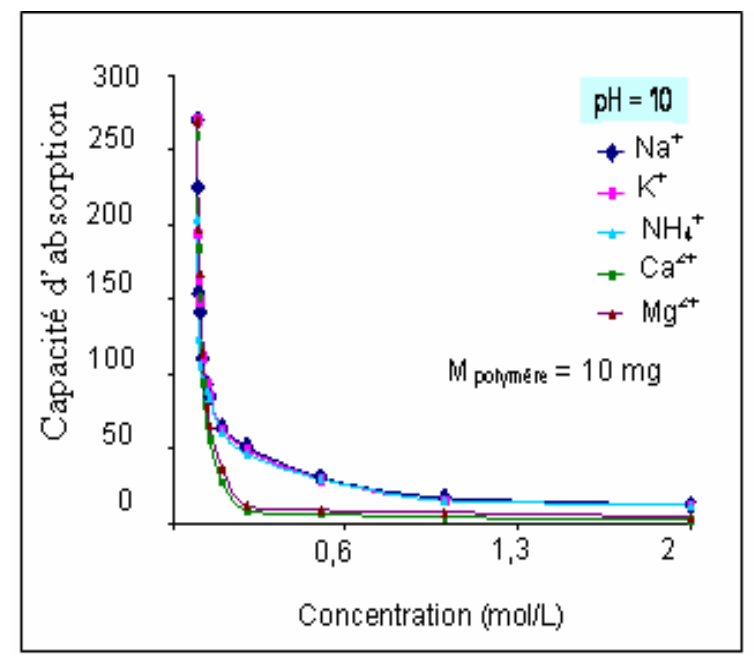

Figure 8 : Variation de la capacité d'absorption du polymère en fonction de la concentration de la solution absorbée à $\mathrm{pH}=10$.

Les cations divalents ont un effet plus accentué par rapport à celui des cations monovalents. En effet, pour les cations divalents, en plus de l'effet d'écran, peut s'ajouter la possibilité de complexation des groupes carboxylates. La formation de complexes inter et intramoléculaires entraîne une réticulation supplémentaire du réseau polymérique et empêchant par voie de conséquence l'expansion de celuici.

\section{CONCLUSION}

La présente étude concerne un polymère superabsorbant capable d'absorber et retenir jusqu'à 300 fois sa masse en eau. L'étude de l'effet de la disposition et du pourcentage $(0,2,0,5$ et $1 \%)$ de ce polymère vis à vis du sol sur le taux de rétention de l'eau par le sol a été effectuée. Les résultats expérimentaux ont montré que la quantité d'eau retenue par le sol en présence du polymère est considérablement supérieure à celle retenue par le sol seul. En effet, la quantité d'eau retenue augmente au fur et à mesure avec l'accroissement du pourcentage du polymère dans le sol. La disposition du polymère peut également influencer le taux de rétention d'eau par le système polymère - sol. En fait, le taux de rétention du système étudie augmente avec la disposition du polymère par 
rapport au sol dans l'ordre suivant : au dessous < mélange $<$ au dessus. Ceci peut être dû à la compacité exercée par le sol sur les chaînes macromoléculaires (lorsque le polymère mis au dessous ou mélangé avec le sol) empêchant par voie de conséquence l'expansion du réseau polymérique. L'évaluation de l'effet de la température sur la capacité d'absorption du polymère a été réalisée pour des températures allant de 10 à $90^{\circ} \mathrm{C}$. A partir d'une température de la solution absorbée de $400^{\circ} \mathrm{C}$, une réduction du taux du gonflement du polymère a été identifiée. Ce résultat peut être attribué à la sensibilité des chaînes polymériques vis-à-vis de la température. En effet, l'élasticité des chaînes peut être affectée sous l'effet de la température, ce qui peut provoquer la libération de l'eau à l'extérieur du réseau. L'étude réalisée a montré aussi que le $\mathrm{pH}$ de la solution absorbée a un effet sur le taux d'absorption du gel. En milieu acide, l'augmentation de la valeur du $\mathrm{pH}$ favorise l'ionisation des groupes acides et par conséquent les répulsions électrostatiques entre sites chargés. Ainsi le taux d'absorption croît au fur et à mesure avec l'augmentation du $\mathrm{pH}$. En milieu basique les cations sodium issus de la soude utilisée pour assurer les valeurs de $\mathrm{pH}$ basiques peuvent faire écran sur les sites chargés et empêcher le gonflement du gel. Un comportement similaire a été observé en évaluant l'effet de la présence des cations mono ou divalents dans le milieu. En fait la présence des contres ions cause une chute drastique du taux d'absorption du gel. Cet effet est plus accentué en présence des cations divalents. Ces derniers peuvent neutraliser plusieurs sites en même temps ainsi qu'ils peuvent former des complexes inter et intramoléculaires avec les groupes carboxylates empêchant ainsi l'expansion du réseau polymérique.

\section{REMERCIEMENTS}

Nos vifs remerciements s'adressent à toutes les personnes qui ont contribué à la réalisation de ce travail.

\section{REFERENCES}

[1] : Peng Chen, Wei'an Zhang, Wei Luo, yue'e Fang, 2004, "Synthesis of superabsorbent polymers by irradiation and their applications in agriculture", J Appl Polym Sci, 93, 1748-1755.

[2] : Erdener Karadag, Dursun Saraydin, Yasmin Caldiran and Olgun Güven, 2000, "Swelling studies of copolymeric Acrylamide/Crotonic Acid hydrogels as Carries for agricultural Uses", Polym Advan Technol, 11, 59-68.

[3] : Bassaid. S, Chaib. M, Bouguelia. A, Trari. M, 2008, "Elaboration and characterization of poly (acrylic acid-cocrotonic acid) copolymers: Application to extraction of metal cations $\mathrm{Pb}(\mathrm{II}), \mathrm{Cd}(\mathrm{II})$ and $\mathrm{Hg}$ (II) by complexation in aqueous Media", React Funct Polym, 68, 483-491.

[4] : P.S. Keshava Murthy, Murali Mohan, J. Sreeramulu, K. Mohana Raju, 2006, "Semi-IPNs of starch and poly(acrylamide-co-Sodium methacrylate): Preparation, swelling and diffusion characteristics evaluation", React Funct Polym, 66, 1482-1493.

[5] : An Li, Jumping Zhang and Aiqin Wang, 2005, "Synthesis, characterization and water absorbency properties of poly(acrylic acid)/ sodium humate superabsorbent composite, Polym Advan Technol, 16, 657680.

[6] : Wen-Fu Lee, Lin-Gi Yang, 2004, "Superabsorbent polymeric materials. XII. Effect of montmorillonite on water absorbency for poly(sodium acrylate) and montmorillonite nanocomposite superabsorbents", J Appl Polym Sci, 92, 3422-3429.
[7] : An Li, Aiqin Wang, 2005, "Synthesis and properties of clay-based Superabsorbent composite", Eur Polym J, 41, 1630-1637.

[8] : Junping Zhang, Ruifeng Liu, An Li, Aiqin Wang, 2006, "Preparation, swelling behaviors and application of polyacrylamide /attapulgite superabsorbent composites" Polym Advan Technol, 17, 12-19.

[9] : Jian Chen, John M. Stark, 2000, "plant species effects and carbon and nitrogen cycling in a sagebrush-crested wheatgrass soil”, Soil Biol Biochem, 32, 47-57.

[10] : Olivier Lambert, Oscar Nagele, Veronique Loux, Jean-Daniel Bonny, Laurent Marchal-Heussler, 2000, "Poly(ethylene carbonate) mocrospheres: manufacturing process and internal structure Characterization" $J$ of Control Release, 67, 89-99.

[11] : Vivek A V, Dhamodharan R, 2008, "Amphiphilic polystyrene-graft-poly(N,N-dimethylamine-2-ethyl methacrylate) hydrogels synthesized via room temperature ATRP:Studies on swelling behaviour and dye sorption", React Funct Polym, 68, 967-973. 